quence of producing a very factual account and the delicate problem of analysis of primitive conditions in a specialized animal has been largely lost in the wealth of information.

In a comprehensive opening chapter, Hubbs and Potter discuss distribution, phylogeny and taxonomy. The remarkable clarity of presentation is slightly marred by small discrepancies in the analytical key for adult lampreys. The typical dentition of the genus Eudontomyzon does not quite conform with the diagram of Eudontomyzon danfordi and for the subgenus Lampetra the shape and distribution of the posterior circumoral teeth are used as diagnostic characters although these teeth are absent. The key is based primarily on dentition, so the problem of ammocoete identification still remains.

The implications of the recently discovered fossil lampreys are discussed by Bardack and Zangerl. But in view of the similarities between this 280 -millionyear-old fossil and modern lampreys it tells us little of the presumed ostracoderm ancestors of lampreys. The treatment of the origin of the lampreys is very brief and could have been greatly extended.

Two chapters by the editors describe the behaviour, ecology and growth of larval lampreys and the general biology of adult animals. Information has been collected from diverse sources to produce an excellent account which will appeal to the general reader as well as the more serious student of lamprey biology.

Perhaps the greatest stimulus to research into lamprey biology has been the disastrous predation by the landlocked sea lamprey in the Great Lakes. In view of the size and urgency of this problem, many of the data have been collected in less controlled conditions than one would expect of a purely scientific investigation. B. R. Smith has performed a difficult task by selecting from the vast body of information and presenting a clear account of the Great Lakes problem.

One of the more recent trends in lamprey evolution has been the elimination of the parasitic phase to produce the so-called "brook lampreys". Hardisty and Potter, in a well written chapter, have paid particular attention to the evolutionary mechanisms involved and interpret brook lamprey speciation as the result of an extension of the larval phase together with changes in the phasing of gonadogenesis in contrast to the traditional explanation of partial paedomorphosis.

In their introduction to a short chapter on the chromosomes Potter and Robinson emphasize the taxonomic significance of the karyotype. In view of the enormous intraspecific variation and the obvious shortage of data, it is surprising that this subject has been treated separately. It would have been more useful had the data been presented in the chapters concerned with taxonomy and evolution (chapters 1, 2 and 6).

The cytological aspects of gonadogenesis are well documented by Hardisty although a simplified diagram of gonadal development, based on data from Lampetra planeri, would have been a distinct advantage. Limited endocrinological knowledge makes conclusions concerning the control of gonadogenesis very tentative. This first volume is completed by Piavis with an account of lamprey embryology.

In summary, this volume is a well presented, extensive review of the evolution and general biology of the lampreys. Many aspects of physiology and endocrinology are covered in volume 2 , and this is in every respect complementary to volume 1 . A book of this standard will be an asset to all libraries catering for students of fish biology or vertebrate zoology in general. Alan D. Pickering

\section{Consequences of Science} l'Engagement Social du Scientifique: Conferences du Cinquantenaire de la Faculté des Sciences, Université de Montreal. By Jean-Jacques Salomon, Andre Des Marais and Jean Dorst. Pp. 99. (Université de Montreal: Montreal, 1971.) \$1.

Politics and Technology. By Roger Williams. Pp. 80. (Macmillan: London, February 1972.) 60p.

l'Engagement contains three addresses delivered at the fiftieth anniversary celebration of the Faculty of Sciences in Montreal University. Salomon discusses the social responsibilities of scientists as they have emerged. He remarks that when Bacon announced that "knowledge is power," he did not mention the difficulties implicit in this association. The idea of pure knowledge remained dominant. By the end of the Second World War the situation had changed fundamentally. In the war scientists associated with statesmen and strategists, and became established at the centre of politics. Science became limited to a sub-autonomy in society. The application of science brought abundance, and this required organization and planning. The quantitative caused a qualitative change.

The nature of modern science and the structure of modern society make them inevitable partners. The scientist's social duty to explain the implications of science to his fellow men, so that the best use can be made of it.

Des Marais discusses the recent evolution of the education of academic scientists in Canada. There is no precedent for the present era, which is the consequence of the scientific explosion. To cope with it, it is necessary to revolutionize traditional notions of the organization of science and society. After a period of very rapid expansion consolidation is necessary. This involves the choice of priorities. These cannot be made without scientists, whose continued participation is now essential for the functioning of society.

Jean Dorst deals with the responsibilities of the ecologist. Many ecologists work in ivory towers. This attitude isolates them from the complicated problems of the biosphere. They should make their views known, and secure the support of the masses. They must inform the political authorities of ecological problems, and the urgency of their solution. They must create an ecological policy. Fie wants a new civilization in which the activities of man and the rest of the living world are carried on harmoniously.

Roger Williams's conclusions are that technology expands the politically possible, and there is a world-wide expectation of continually improving material standards. If technocracy is the newest mode of government, the older characteristics of muddle, incompetence, insincerity, injustice, and barbarity remain. Yet technological society is likely to seem increasingly pleasant to most of its members.

Williams has quoted 263 authorities in 54 pages. If his conclusions are not inspiring, he has provided students with a useful annotated bibliography.

\section{J. G. CROWTHER}

\section{Separating Cells}

Separations with Zonal Rotors. Edited by Eric Reid. (Wolfson Bioanalytical Centre of the University of Surrey, 1971.) (Distributed by the University of Surrey Bookshop, Guildford, Surrey.) $£ 2.40$.

SCIENTIFIC discoveries progress by leaps and bounds. The reason is generally the development of a new technique, of which the use of zonal centrifugation to separate biological materials is a case in point. The main advantages of zonal rotors over conventional types are that wall effects are avoided and that larger samples can be handled. This booklet is aimed both at the worker already engaged in zonal centrifugation and at the Ininitiated not yet acquainted with the technique.

The first objective is undoubtedly achieved. The forty articles represent contributions from participants at a symposium devoted to this topic. There is a host of information, from 